\title{
Indicadores de bienestar subjetivo de personas con discapacidad, sus familiares y profesionales que trabajan con personas con discapacidad
}

\author{
Indicators of subjective well-being of people with disabilities, their families \\ and professionals who work with people with disabilities \\ Daniel Sojuel (iD *, Donald González-Aguilar (iD , Evelyn Rodríguez-Cisneros \\ Escuela de Ciencias Psicológicas Universidad de San Carlos de Guatemala \\ *Autor al que se dirige la correspondencia: dsojuel@psicousac.edu.gt
}

\section{Resumen}

Recibido: 7 de febrero 2020 / Revisión: 17 de febrero 2021 / Aceptado: 5 de agosto 2021

\begin{abstract}
$\mathrm{L}$ a mayoría de literatura sobre bienestar subjetivo (BS) no está centrada en personas con discapacidad, lo cual

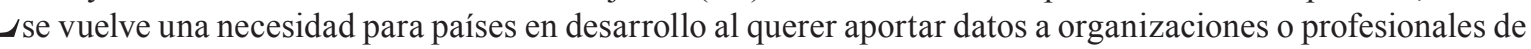
la psicología para la toma decisiones basadas en evidencia en sus iniciativas o práctica profesional. El objetivo de este artículo es presentar, con un alcance descriptivo, la triangulación de indicadores cuantitativos y cualitativos del BS de personas con discapacidad, familiares y trabajadores de organizaciones de y para personas con discapacidad en el departamento de Sololá, Guatemala. El enfoque de la investigación fue mixto. Se recolectó información a través de una encuesta sociodemográfica, la Escala de Satisfacción Con la Vida (SWLS), la Escala de Afecto Positivo y Negativo (SPANAS), además una entrevista semiestructurada. Se evaluó 84 personas, 19 personas con discapacidad, 32 familiares de personas con discapacidad y 33 trabajadores. Las Escalas SWLS $(\omega$ de McDonald $=.7)$ y SPANAS $(\omega$ de McDonald $=.8$ ) tienen un nivel aceptable de confiabilidad. La satisfacción con la vida correlacionó negativamente con el afecto negativo, pero la fuerza fue débil $\left(r_{s}=-.28, \mathrm{n}=97\right.$, $p=.009)$. El $76 \%$ de la muestra se encontró en un nivel muy alto de satisfacción con la vida y $21 \%$ refirió un alto estado afectivo negativo. En el análisis cualitativo, se estableció que, la participación en actividades productivas o recreativas, la convivencia entre redes de apoyo y el cumplimiento de metas personales son situaciones que aportan en el bienestar subjetivo.
\end{abstract}

Palabras claves: Calidad de vida, satisfacción con la vida, bienestar psicológico, apoyo social, inclusión social

\section{Abstract}

$\mathrm{M}$ ost of the literature on subjective well-being (SWB) is not focused on people with disabilities, which becomes a necessity for developing countries when they want to provide data to organizations or psychology professionals to make evidence-based decisions in their initiatives or professional practice. The aim of this study is to present, with a descriptive scope, the triangulation of quantitative and qualitative indicators of the SWB of people with disabilities, family members and workers of organizations of and for people with disabilities in the department of Sololá, Guatemala. The research was done with a mixed method approach. Data was collected through a sociodemographic survey, the Satisfaction With Life Scale (SWLS), the Positive and Negative Affect Scale (SPANAS), and a semi-structured interview. The sample consisted of 84 people were evaluated, 19 people with disabilities, 32 relatives of people with disabilities and 33 workers. The SWLS (McDonaldís $\omega=.7$ ) and SPANAS (McDonaldís $\omega=.8$ ) scales have an acceptable level of reliability. Satisfaction with life was negatively correlated with negative affect, but strength was weak $\left(r_{s}=-.28, \mathrm{n}=97, p=.009\right)$. Also, $76 \%$ of the sample had a very high level of satisfaction with life and $21 \%$ reported a high negative affective state. In the qualitative analysis, it was established that participation in productive or recreational activities, coexistence between support networks and the fulfillment of personal goals are situations that contribute to subjective well-being.

Keywords: Quality of life, satisfaction with life, psychological well-being, social support, social inclusion 


\section{Introducción}

A través de una encuesta poblacional de discapacidad, en 2016 se estableció para la región suroeste de Guatemala, en la que se encuentra el departamento de Sololá, una prevalencia de $10.4 \%$ de discapacidad (Centro Internacional para Evidencia de Discapacidad [ICED], 2016). A partir de la base de datos del Censo de población realizado en 2018 en Guatemala (Instituto Nacional de Estadística [INE], 2020), simulando los criterios de inclusión para la prevalencia utilizados por ICED (2016) a excepción de la evaluación física, se establece una prevalencia de $8.8 \%$ a nivel nacional, $8.8 \%$ en la región suroeste y $7.7 \%$ en Sololá. Este dato de prevalencia se ubica en el límite inferior de los intervalos de confianza brindados por ICED (2016).

En este contexto, en Guatemala, organizaciones de sociedad civil en cooperación con instituciones del estado, realizan distintas acciones en pro de las personas con discapacidad, principalmente para garantizar la igualdad de oportunidades de participación en sociedad y concientizar a la población en general sobre la discapacidad, pero también sobre la importancia de la salud mental.

Estas acciones son fundamentales para justificar la investigación sobre la salud mental de aquellas personas que conforman las redes sociales de apoyo para las personas con discapacidad, donde se encuentran las personas con discapacidad, sus familiares y trabajadores de organizaciones de y para personas con discapacidad.

En el estudio de la salud mental, a partir del siglo 20 el énfasis fue más allá de la patología, pues la medición de la felicidad, tratada en términos de bienestar subjetivo ha adquirido gran relevancia (Diener et al., 2009).

El bienestar subjetivo (BS) es definido como la evaluación general que las personas hacen sobre sus vidas y experiencias emocionales (Diener et al., 1999). El bienestar subjetivo se refiere a las percepciones de las personas sobre lo que constituye una calidad de vida buena o mejorada, y el grado en el que las personas están satisfechas de que son capaces de alcanzar los objetivos que creen que son necesarios para llevar una buena vida (Roelen \& Camfiel, 2015).

El BS tiene dos componentes, uno cognitivo y uno afectivo. El componente cognitivo tiene que ver con la satisfacción con la vida, esta se entiende desde un proceso de introspección, donde el individuo evalúa la calidad de su vida, con base en sus propios criterios, esta generalización de la satisfacción permite que la persona puede evaluar su vida en las áreas que considere más significativas para referir el grado de satisfacción, pero adicional a esto la satisfacción con la vida también puede tomar aspectos del pasado de la persona y del presente, por último la cultura de las personas también puede verse reflejada en la satisfacción con la vida (Pavot \& Diener, 1993).

El componente afectivo del BS tiene que ver con el afecto positivo y negativo. El afecto positivo refleja la medida en que una persona siente ganas de vivir, más que no sentirse angustiado, el afecto positivo requiere una participación activa y entusiasta en la vida cotidiana. El afecto positivo alto se define por palabras tales como excitado, fuerte y eufórico, que expresa energía y compromiso placentero, mientras que el afecto negativo alto representa la medida en que una persona se siente molesta, angustiada, nerviosa, culpable o tensa (Watson y colaboradores 1988).

El estudio del bienestar subjetivo con personas con discapacidad, familiares y trabajadores de organizaciones de y para personas con discapacidad es necesario por tres razones basadas en propuestas de Diener y colaboradores (2009): (a) primero por los beneficios de niveles altos de bienestar subjetivo; (b) porque el bienestar subjetivo es un indicador de la calidad de vida, junto a otros indicadores económicos y sociales, además de ello, en el campo académico es información importante para evaluar a diferentes grupos con características específicas; (c) por último, su estudio es ampliamente necesario para aportar evidencia científica para los múltiples programas en pro de las personas con discapacidad en la región Centroamericana.

El objetivo de este artículo es presentar, con un alcance descriptivo, la triangulación de indicadores cuantitativos y cualitativos del BS de personas con discapacidad, familiares y trabajadores de organizaciones de y para personas con discapacidad en el departamento de Sololá, Guatemala. Esta propuesta es un acercamiento relevante a la investigación en psicología y discapacidad y los resultados serán útiles para aportar en la toma de decisiones basadas en evidencia desde instituciones no gubernamentales o gubernamentales enfocadas en discapacidad. Por otro lado, el sistema de recolección de datos que se describirá en el apartado de Materiales y Métodos puede seguir siendo usado para futuras investigaciones, especialmente útiles para la ruralidad de Guatemala, pero también para otros contextos, como centros de práctica psicológica para apoyar en la evaluación de pacientes o en la docencia en el aprendizaje de estrategias de recolección de datos en psicología. 


\section{Materiales y Métodos}

La investigación, buscando profundizar e integrar diferentes fuentes de información, tuvo un enfoque mixto desde el método no experimental para el área cuantitativa y el método de la teoría fundamentada para el área cualitativa. Se realizó un muestreo no probabilístico, basado en la disponibilidad de los participantes. Se conformó la muestra con 84 personas de cinco organizaciones que realizan trabajo integral (salud, educación y área laboral) con personas con discapacidad en el departamento de Sololá, las cuales forman parte de la Asociación Coordinadora de Organizaciones de y Para Personas con Discapacidad (Acopedis): (a) Senderos de Maíz, ubicada en el municipio de Panajachel, (b) Asopadis, ubicada en San Lucas Tolimán, (c) Centro Maya, ubicada en San Juan la Laguna, (d), Somos hijos del Lago, ubicada en San Pedro la Laguna, y (e) Adisa, ubicada en Santiago Atitlán. Se recopiló información de tres grupos en cada organización: personas con discapacidad que son usuarios de algunos de los servicios ofrecidos, familiares de personas con discapacidad y trabajadores de las organizaciones (salud, educación y administrativos).

\section{Instrumentos}

\section{Encuesta sociodemográfica}

Se recopiló la información de las siguientes variables: municipio, organización a la que pertenece, tipo de discapacidad (para personas con discapacidad y familiares), tipo de parentesco con la persona con discapacidad, sexo, edad, autoidentificación étnica, comunidad lingüística y nivel académico.

\section{Escala de Satisfacción con la Vida (SWLS}

La escala de Satisfacción con la vida, desarrollada por Diener y colaboradores (1985) es un instrumento unifactorial que consta de cinco afirmaciones que refieren a la valoración cognitiva del bienestar subjetivo: "En la mayoría de los sentidos mi vida se acerca a mi ideal"; "Las condiciones de mi vida son excelentes"; "Estoy satisfecho/a con mi vida"; "Hasta ahora he conseguido las cosas importantes que quiero en la vida"; "Si tuviera que vivir mi vida de nuevo, no cambiaría casi nada". Las afirmaciones se calificaron en una escala Likert de cinco puntos siguiendo las recomendaciones de Kobau, Sniezek, Zack, Lucas y Burns (2010): 1, "Totalmente de acuerdo; 2, "Algo en desacuerdo"; 3, "Ni de acuerdo, ni en desacuerdo"; 4, Algo de acuerdo"; 5, "Totalmente de acuerdo". Los puntajes de cada ítem se suman para tener un total que varía de cinco a 25 puntos. Los puntajes totales se pueden clasificar de la siguiente manera: Muy alta satisfacción con la vida (20-25 puntos); Alta satisfacción con la vida (15-19 puntos); Ni baja ni alta satisfacción con la vida (10-14 puntos); y Baja satisfacción con la vida (5-9 puntos).

\section{Escala de Afecto Positivo y Negativo (SPANAS)}

Se utilizó la versión en español de la Escala de Afecto Positivo y Negativo (SPANAS) desarrollada por (Joiner et al., 1997). Esta escala representa la valoración afectiva del bienestar subjetivo. Cuenta con 20 palabras, 10 que representan sentimientos y emociones positivas y 10 negativas. Cada participante debía responder la intensidad de la experiencia de los 20 sentimientos y emociones con un marco de referencia de un mes hacia atrás. Las respuestas se calificaron en una escala Likert de cinco puntos: 1, "Levemente o casi nada"; 2, "Un poco"; 3, "Moderadamente"; 4, "Bastante"; 5, "Extremadamente". Al realizar la sumatoria de los ítems de cada escala por separado (en la Tabla 1 se observan los ítems correspondientes a cada escala), se pueden interpretar de la siguiente manera: Muy alto afecto positivo o negativo (41-50 puntos); Alto afecto positivo o negativo (31-40 puntos); Ni bajo, ni alto afecto positivo o negativo (21-30 puntos); Bajo afecto positivo o negativo (10-20).

Se hizo modificaciones a algunas palabras de la escala para su mejor comprensión, además se incluyó una tabla para los acompañantes y traductores con el fin de manejar las mismas definiciones para cada palabra y hacer la diferenciación de palabras como asustado y atemorizado que tienen una traducción e interpretación muy similar en idiomas mayas y lengua de señas. Estas definiciones se pueden observar en la Tabla 1. Las palabras que fueron ajustadas fueron: "orgulloso" por "orgulloso de usted mismo", ya que esta daba a entender arrogancia en vez de orgullo como una valoración positiva. También se cambió "estimulado" por "emocionado" porque se considera una traducción de "Excited" mejor entendida en el contexto, misma razón por la que se cambió "Miedoso" por "Intranquilo" al ser una mejor traducción en el contexto de "Jittery". 
Tabla 1

Definiciones de Escala de Afecto Positivo y Negativo (SPANAS)

\begin{tabular}{|c|c|c|}
\hline Ítem & Escala correspondiente & Definición \\
\hline Interesado & Afecto positivo & $\begin{array}{l}\text { Refleja el interés por la vida, por las actividades que se realizan y } \\
\text { por lo que hay alrededor. }\end{array}$ \\
\hline Irritable & Afecto negativo & $\begin{array}{l}\text { Refleja la facilidad en que cualquier situación haga sentirse "irrita- } \\
\text { do" a la persona. }\end{array}$ \\
\hline Entusiasmado & Afecto positivo & Tener o mostrar goce intenso ante un hecho o situación en la vida. \\
\hline Tenso con malestar & Afecto negativo & $\begin{array}{l}\text { Sentimiento físico relacionado al estrés, regularmente se ubica en } \\
\text { el cuello, pero no exclusivamente. }\end{array}$ \\
\hline Disgustado, molesto & Afecto negativo & Enojado por alguna situación vivida \\
\hline Fuerte; enérgico & Afecto positivo & $\begin{array}{l}\text { Estar lleno de energía en las actividades, o tener las fuerzas para } \\
\text { trabajar o hacer diferentes actividades. También puede verse como } \\
\text { una forma de sentirse, aunque no se tengan actividades que requi- } \\
\text { eran de fuerza física. }\end{array}$ \\
\hline $\begin{array}{l}\text { Orgulloso de usted } \\
\text { mismo }\end{array}$ & Afecto positivo & $\begin{array}{l}\text { Sentimiento positivo de haber logrado un objetivo o de estar feliz } \\
\text { con uno mismo, muy diferente de ser orgulloso sinónimo de "ser } \\
\text { creído". }\end{array}$ \\
\hline $\begin{array}{l}\text { Temeroso, atemo- } \\
\text { rizado }\end{array}$ & Afecto negativo & $\begin{array}{l}\text { Miedo que se siente al considerar que algo perjudicial o negativo } \\
\text { ocurra o haya ocurrido. Preocupado de que algo indeseable ocur- } \\
\text { rirá o se hará. }\end{array}$ \\
\hline Avergonzado & Afecto negativo & Sentimientos de vergüenza. \\
\hline Inspirado & Afecto positivo & $\begin{array}{l}\text { Animado, o lleno de las ganas de hacer algo, como si fuera dado } \\
\text { por algo divino, alguien especial o la persona misma. }\end{array}$ \\
\hline Nervioso & Afecto negativo & Alterarse o irritarse por una cosa o con una persona. \\
\hline Emocionado & Afecto positivo & Sentimiento positivo causado por alguna situación. \\
\hline Decidido & Afecto positivo & Que la persona se siente firme y segura de algo. \\
\hline Atento & Afecto positivo & De poner atención cuando se necesita. \\
\hline Intranquilo & Afecto negativo & Que no tiene tranquilidad física o interna. \\
\hline Activo & Afecto positivo & Que realiza muchas actividades, que se es productivo. \\
\hline Asustado & Afecto negativo & $\begin{array}{l}\text { Impresión momentánea de miedo causada por algo que aparece u } \\
\text { ocurre de forma repentina e inesperada y que generalmente altera o } \\
\text { agita la respiración. }\end{array}$ \\
\hline Culpable & Afecto negativo & Que la persona siente culpa por alguna situación de su vida. \\
\hline Alerta, despierto & Afecto positivo & Situación de vigilancia, atención constante alrededor. \\
\hline Hostil & Afecto negativo & Agresivo, enojado o antipático \\
\hline
\end{tabular}




\section{Cuestionario para entrevista semiestructurada}

Relacionado al bienestar subjetivo, se incluyeron distintas preguntas para cada grupo. Para las personas con discapacidad se incluyeron las categorías: (a) relación del apoyo por parte de familiares, amigos y trabajadores de las organizaciones con el bienestar personal, y (b) relación de participación en alguno de los servicios que brindan las organizaciones con el bienestar personal. Para los familiares se incluyó preguntas relacionadas a la forma en que el apoyo recibido por las organizaciones repercute en la familia y para los trabajadores de las organizaciones se preguntó sobre cómo el trabajo que realizan se relaciona con su bienestar.

\section{Procedimiento}

Se realizó tres tipos de traducción a los instrumentos utilizados, una al español únicamente para la escala SWLS, y para las escalas SPANAS y SWLS se realizaron traducciones al tz'utujil y al kaqchikel (Apéndices A al D en archivos complementarios), esto se realizó para garantizar la inclusión de participantes en la muestra con un idioma materno distinto al español. Con el apoyo de la Tabla 1, estas traducciones se realizaron con el apoyo de dos maestros con dominio de escritura del tz'utujil y dos personas del equipo de Acopedis en la revisión del tz'utujil y kaqchikel. Se realizaron los ajustes que estas personas solicitaron en los instrumentos. Por otro lado, se trabajó con cuatro mujeres profesionales en la interpretación a lengua de señas de los instrumentos a utilizar, cada una llevó un proceso de familiarización con los instrumentos en español para tener una estructura básica de la interpretación en lengua de señas que se adaptó a las dudas que presentaron los participantes al momento de la recopilación de información.

Para la recopilación de información se utilizó la técnica de entrevista personal asistida por ordenador (CAPI) con el software CSpro. Se programaron los instrumentos en idioma español, tz'utujil y kaqchikel para ser aplicados en tablets con sistema operativo Android. También se elaboró material de apoyo para responder y recordar el significado de la escala Likert de 1 al 5 para la escala SPanas y 1 al 7 en la escala SWLS. Este material consistió en un cartel resistente, con imágenes de cinco termómetros con distinta intensidad que reflejaba cada una de las opciones de respuesta.
Con las herramientas listas, se procedió a la realización de una prueba piloto con 10 personas para hacer ajustes al sistema de captura, esto dio resultado cambios en las traducciones de los ítems y en la escala SWLS hacia una medición de 1 a 5 para igualar la forma de respuesta en ambas escalas.

Posterior a los ajustes necesarios, con un encargado designado de cada organización de las cinco seleccionadas, se hicieron citas en hogares o se estableció un día para recibir a las personas en las instalaciones estas para completar la recopilación de información.

\section{Procesamiento y análisis de datos}

Con el uso del software CSPro, la captura de datos tuvo una estructura de hogares, es decir se registró en un mismo grupo, la información de familiares y personas con discapacidad, esto con motivos de futuros análisis, de la misma forma se registró la información de trabajadores según la organización a la que pertenecen. Se llevó a cabo un proceso de limpieza de la base de datos, para eliminar registros en blanco creados por la manipulación del sistema en el campo y la corrección de inconsistencias en el llenado de información demográfica. Se hizo la exportación de dos bases de datos a formato ".csv" para proceder con el análisis de datos con el software SPSS, el cual comenzó con la concatenación de ambas bases de datos, una correspondiente a las personas con discapacidad y familiares y la otra a trabajadores de las organizaciones.

Se generó una tabla compuesta con los resultados descriptivos de las escalas utilizadas, desagregando la información según el tipo de participante en el estudio. Para cada escala evaluada se hizo un cálculo de normalidad de los datos recabados con la prueba de Shapiro Wilk; se utilizó la prueba Omega ( $\omega)$ de McDonald, estableciendo un punto de corte de .7 para determinar la confiabilidad de los datos; se calculó la medida de adecuación muestral de Kaiser-Myer-Olkin como indicador mínimo de validez de constructo, además de una correlación de Spearman entre las escalas evaluadas para determinar la validez convergente; por último, se hizo una comparación de los resultados de ambas escalas evaluadas con la prueba Kruskal-Wallis entre tipo de participantes. En las pruebas de hipótesis se estableció un nivel de significancia de .05.

Para la información cualitativa, se utilizó el software Nvivo12 Pro, realizando análisis de vídeo directamente, generando categorías y memos analíticos. 
Se seleccionaron temas de interés con la temporalidad especificada en cada video, para transcribir extractos de las entrevistas con fines de análisis y discusión de resultados.

\section{Consideraciones éticas}

Se utilizó un consentimiento informado para los participantes. Se aclaró que la participación en el estudio era completamente voluntaria, sin existir ningún tipo de consecuencia desfavorable en caso de no aceptar participar. Se indicó que era posible retirarse en el momento que la persona desee. Se aseguró que la participación en el estudio no significaba ningún gasto. En el transcurso de la entrevista los participantes tenían la oportunidad de solicitar información actualizada sobre el estudio a los investigadores responsables. Se solicitó el permiso de grabar la sesión en video para poder transcribir la información relevante para el análisis. Se aseguró la confidencialidad de la información recabada, no siendo usada para ningún otro propósito fuera de los de la investigación, se aclaró que no se revelaría la identidad de forma directa de los participantes. Una vez transcritas las entrevistas, el archivo con el audio de las grabaciones se destruirá después de dos años a partir de la entrevista. Se indicó a los participantes que, al preferir no firmar el documento, su consentimiento para participar en el estudio podía ser grabado.

\section{Resultados}

\section{Descripción de la muestra}

La muestra se conformó por 33 trabajadores de las organizaciones (12 de salud, 12 de educación, 5 directivos y 5 administrativos); 19 personas con discapacidad y 32 familiares de personas con discapacidad. La edad de los trabajadores estuvo en un rango de 21 a 63 años $(M=32, S D=10)$; el rango de edad para para personas con discapacidad fue de 18 a 31 años $(M=25, S D=5)$; y para familiares de personas con discapacidad fue de 18 a 78 años $(M=45, S D=15)$. Se presentan las características de la muestra con detalle en la Tabla 2 y en la Tabla 3 se presentan estadísticos descriptivos para cada una de las escalas evaluadas, desagregadas por tipo de participante.

\section{Confiabilidad y validez de los instrumentos}

Según la prueba Shapiro Wilk, se encontró que los datos para la escala SWLS no son normales $(p<$ $.001)$, mientras que los datos para la escala de afecto positivo fueron normales ( $p=.2$ ), el mismo resultado se encontró para la escala de afecto negativo $(p=.08)$.

Se encontró que la Escala de Satisfacción con la Vida (SWLS) es confiable en un nivel aceptable (5 ítems; $\omega$ de McDonald = .7) y la Escala de Afecto Positivo y Negativo (Spanas) es confiable en un nivel bueno (20 ítems; $\omega$ de McDonald = .8). En cuanto a las subesbalas de Spanas, la escala de afecto positivo resultó confiable en un nivel aceptable (10 ítems; $\omega$ de McDonald $=.7)$ y la escala de afecto negativo resultó confiable en un nivel bueno (10 ítems; $\omega$ de McDonald = .8).

Para la escala SWLS, el análisis de componentes principales para esta escala demostró que los cinco ítems corresponden a un solo componente, tal y como se propone en la escala utilizada. La medida de adecuación muestral de Kaiser-Meyer-Olkin, con una rotación oblim, indicó que la fuerza de las relaciones entre las variables fue regular $(K M O=.7$;), esto junto a los resultados de la prueba de esfericidad de Bartlett $\left(\chi^{2}(10, \mathrm{~N}=97)=58.97 p<.001\right)$ permite asegurar que los resultados del instrumento se puedan someter a un análisis factorial, esto para brindar mayor robustez al hallazgo de que la escala está compuesta por un único componente, el cual entonces podrá ser entendido como único factor (satisfacción con la vida).

También, la fuerza de las relaciones entre las variables para la escala Spanas fue regular $(K M O=.7)$ y la prueba de esfericidad de Bartlett resultó significativa $\left(\chi^{2}(190, \mathrm{~N}=97)=549.79 p<.001\right)$. Al especificar la extracción de dos componentes en el análisis de componentes principales, los ítems que corresponden a cada escala se posicionan correctamente según afecto positivo y negativo.

Como indicador de validez convergente se realizó una correlación de Spearman entre la escala SWLS con cada una de las subescalas de Spanas y se observó que la satisfacción con la vida correlaciona negativamente con el afecto negativo, pero la fuerza es débil $\left(r_{S}=-.28, \mathrm{n}=97, p=.009\right)$. Los resultados según los puntos de corte para ambas escalas se pueden observar en la Tabla 4 en donde se desagregan según sexo, autoidentificación étnica y el tipo de participante. 
Tabla 2

Características de la muestra

\begin{tabular}{|c|c|c|c|c|c|c|c|c|}
\hline \multirow[t]{2}{*}{ Características de la muestra } & \multicolumn{2}{|c|}{ Profesionales } & \multicolumn{2}{|c|}{$\begin{array}{l}\text { Personas con } \\
\text { discapacidad }\end{array}$} & \multicolumn{2}{|c|}{ Familiares } & \multicolumn{2}{|c|}{ Total } \\
\hline & $\mathrm{f}$ & $\%$ & $\mathrm{f}$ & $\%$ & $\mathrm{f}$ & $\%$ & $\mathrm{f}$ & $\%$ \\
\hline Número de participantes & 33 & 100 & 19 & 100 & 32 & 100 & 84 & 100 \\
\hline \multicolumn{9}{|l|}{ Municipio } \\
\hline Sololá & 0 & 0 & 1 & 5 & 1 & 3 & 2 & 2 \\
\hline Panajachel & 7 & 21 & 5 & 26 & 5 & 16 & 17 & 20 \\
\hline San Antonio Palopó & 0 & 0 & 2 & 11 & 2 & 6 & 4 & 4 \\
\hline San Lucas Tolimán & 4 & 12 & 3 & 16 & 7 & 22 & 14 & 19 \\
\hline San Juan la Laguna & 5 & 15 & 2 & 11 & 2 & 6 & 9 & 9 \\
\hline San Pedro la Laguna & 6 & 18 & 1 & 5 & 3 & 9 & 10 & 12 \\
\hline Santiago Atitlán & 11 & 33 & 5 & 26 & 12 & 38 & 28 & 34 \\
\hline \multicolumn{9}{|l|}{ Organización a la que pertenece } \\
\hline Adisa & 11 & 33 & 5 & 26 & 12 & 38 & 28 & 34 \\
\hline Asopadis & 4 & 12 & 5 & 26 & 9 & 28 & 18 & 23 \\
\hline Senderos de maíz & 6 & 18 & 6 & 32 & 6 & 19 & 18 & 21 \\
\hline Centro maya & 5 & 15 & 2 & 11 & 2 & 6 & 9 & 9 \\
\hline Somos hijos del lago & 6 & 18 & 1 & 5 & 3 & 9 & 10 & 12 \\
\hline Acopedis & 1 & 3 & 0 & 0 & 0 & 0 & 1 & 1 \\
\hline \multicolumn{9}{|l|}{ Sexo } \\
\hline Hombre & 6 & 18 & 11 & 58 & 4 & 13 & 21 & 27 \\
\hline Mujer & 27 & 82 & 8 & 42 & 28 & 88 & 63 & 73 \\
\hline \multicolumn{9}{|l|}{ Autoidentificación étnica } \\
\hline Maya & 23 & 70 & 13 & 68 & 27 & 84 & 63 & 75 \\
\hline Mestizo & 2 & 6 & 2 & 11 & 2 & 6 & 6 & 7 \\
\hline Ladino & 6 & 18 & 4 & 21 & 3 & 9 & 13 & 15 \\
\hline No declarado & 2 & 6 & 0 & 0 & 0 & 0 & 2 & 2 \\
\hline \multicolumn{9}{|l|}{ Comunidad lingüística } \\
\hline Tz'utujil & 15 & 45 & 7 & 37 & 15 & 47 & 37 & 44 \\
\hline Kaqchikel & 3 & 9 & 4 & 21 & 8 & 25 & 15 & 18 \\
\hline Kícheí & 2 & 6 & 0 & 0 & 0 & 0 & 2 & 2 \\
\hline Qíeqchií & 0 & 0 & 0 & 0 & 0 & 0 & 0 & 0 \\
\hline Otro & 0 & 0 & 2 & 11 & 1 & 3 & 3 & 3 \\
\hline No aplica & 11 & 33 & 6 & 32 & 8 & 25 & 25 & 33 \\
\hline No declarado & 2 & 6 & 0 & 0 & 0 & 0 & 2 & 2 \\
\hline
\end{tabular}


Tabla 2 (Continuación)

\begin{tabular}{|c|c|c|c|c|c|c|c|c|}
\hline \multirow[t]{2}{*}{ Características de la muestra } & \multicolumn{2}{|c|}{ Profesionales } & \multicolumn{2}{|c|}{$\begin{array}{l}\text { Personas con } \\
\text { discapacidad }\end{array}$} & \multicolumn{2}{|c|}{ Familiares } & \multicolumn{2}{|c|}{ Total } \\
\hline & $\mathrm{f}$ & $\%$ & $\mathrm{f}$ & $\%$ & $\mathrm{f}$ & $\%$ & $\mathrm{f}$ & $\%$ \\
\hline \multicolumn{9}{|l|}{ Nivel académico } \\
\hline Ninguno & 0 & 0 & 1 & 5 & 11 & 34 & 12 & 12 \\
\hline Preprimaria & 0 & 0 & 2 & 11 & 3 & 9 & 5 & 9 \\
\hline Primaria & 1 & 3 & 5 & 26 & 7 & 22 & 13 & 20 \\
\hline Básico & 1 & 3 & 3 & 16 & 3 & 9 & 7 & 8 \\
\hline Diversificado & 11 & 33 & 7 & 37 & 7 & 22 & 25 & 26 \\
\hline Licenciatura & 20 & 61 & 1 & 5 & 1 & 3 & 22 & 23 \\
\hline Maestría & 0 & 0 & 0 & 0 & 0 & 0 & 0 & 0 \\
\hline No declarado & 0 & 0 & 0 & 0 & 0 & 0 & 0 & 2 \\
\hline \multicolumn{9}{|l|}{ Tipo de discapacidad $^{\mathrm{a}}$} \\
\hline Total & - & - & 19 & 100 & 32 & 100 & 51 & 100 \\
\hline Visual & - & - & 5 & 26 & 6 & 19 & 11 & 22 \\
\hline Auditiva & - & - & 8 & 42 & 11 & 34 & 19 & 37 \\
\hline De movimiento & - & - & 6 & 32 & 15 & 47 & 21 & 41 \\
\hline \multicolumn{9}{|c|}{ Parentesco con personas con discapacidad } \\
\hline Total & - & - & - & - & - & - & 32 & 100 \\
\hline Madre/padre & - & - & - & - & - & - & 26 & 81 \\
\hline Hermana/hermano & - & - & - & - & - & - & 3 & 9 \\
\hline Otra persona familiar & - & - & - & - & - & - & 3 & 9 \\
\hline
\end{tabular}

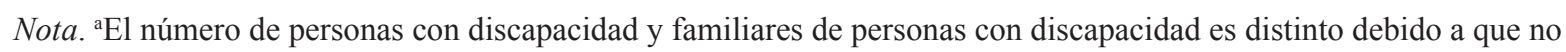
se logró entrevistar a algunas personas de ambos grupos. 


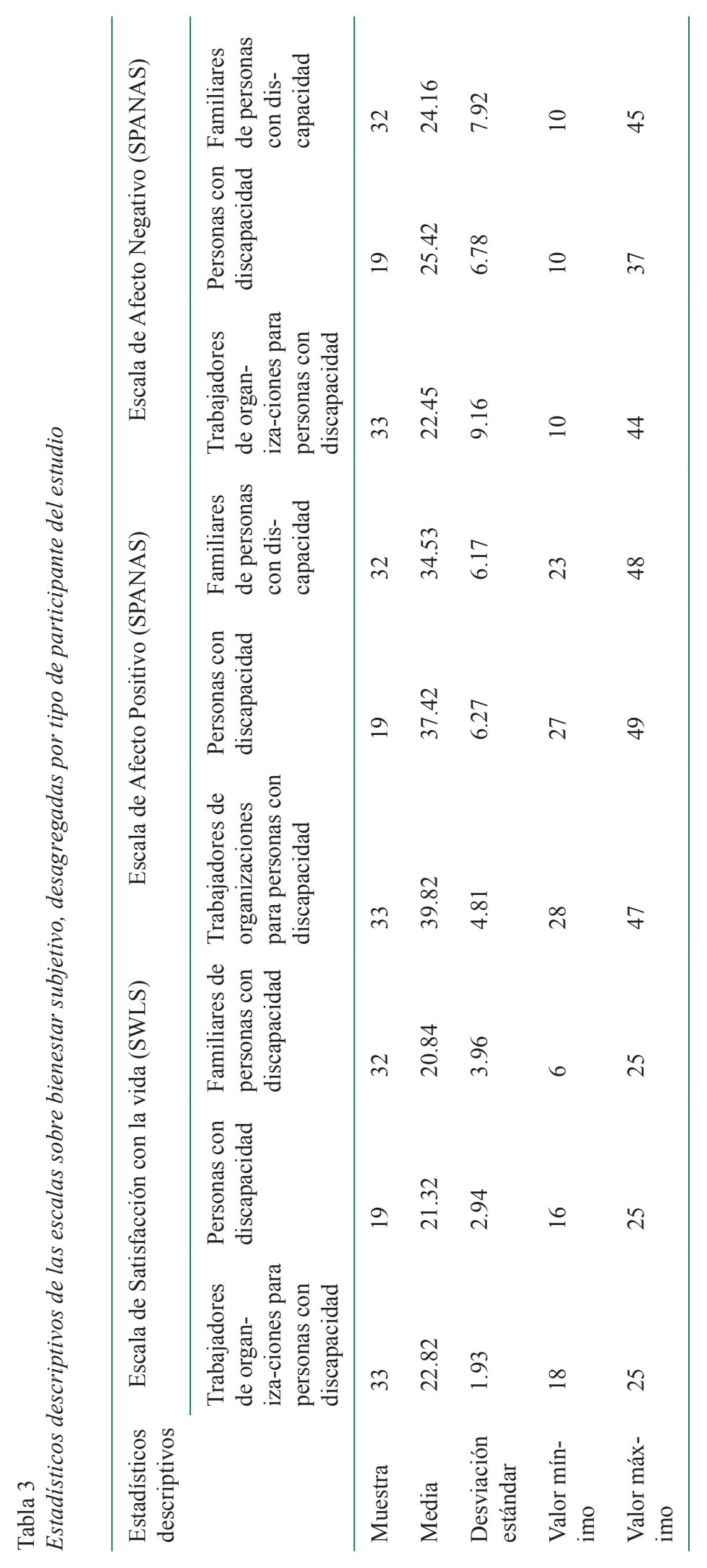




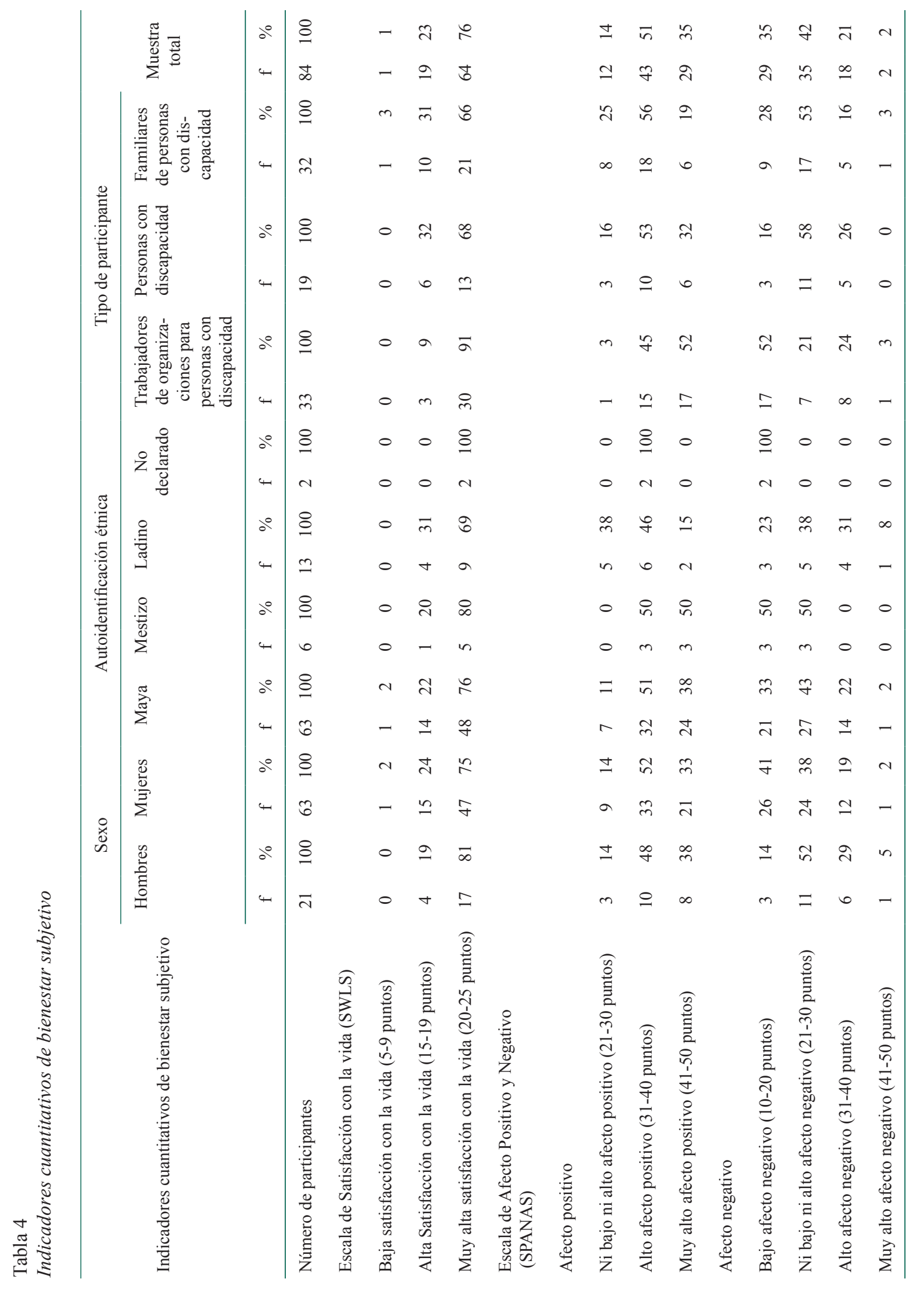


También se realizó una comparación entre participantes en la satisfacción con la vida y el afecto positivo y negativo con la prueba Kruskall Wallis. Solo se encontró diferencia significativa en el afecto positivo entre los participantes del estudio, $\chi^{2}(2)=12.5$, $p=.002$, con un rango medio de 52.97 para los trabajadores de las organizaciones, 31.61 para familiares de personas con discapacidad y 42.66 para personas con discapacidad.

\section{Indicadores cualitativos de bienestar subjetivo}

Para las personas con discapacidad, la categoría "relación del apoyo por parte de familiares, amigos y trabajadores de las organizaciones con el bienestar personal", se puede detallar con los siguientes hallazgos a partir de la agrupación de respuestas a cada una de las preguntas de la entrevista: (a) existe un reconocimiento de que la familia brinda sustento emocional, compañía y amistad; y la organización brinda servicios de rehabilitación que permiten el alcance de mayor satisfacción con la vida; (b) a quien le dan mayor confianza, el verdadero amigo o amiga, es quien los trata con dignidad y por eso algunas personas refieren tener pocos amigos, en este sentido, se encuentra que un trato condescendiente afecta negativamente en el bienestar que, pues da el mensaje "tú no sabes pensar", y para los adultos un mensaje de "eres un niño o niña"; (c) salir a la calle, tener nuevas experiencias en sus comunidades afecta positivamente el bienestar; (d) cuando se indaga con profundidad sobre las creencias que tienen de sí mismos, es muy posible encontrar una lucha con la idea del empoderamiento, con la idea de los propios derechos y de ser parte de la diversidad humana, y también refieren que constantemente tratan de pensar positivo con el apoyo familiar y de las organizaciones, pero que en ocasiones estos pensamientos no pueden mantenerse y prevalecen estados emocionales negativos.

Con relación a la categoría "relación de participación en alguno de los servicios que brindan las organizaciones con el bienestar personal" las personas con discapacidad refieren que: (a) una fuente de bienestar de personas involucradas en algún proceso educativo es la percepción de apoyo en actividades académicas; (b) la convivencia entre personas con el mismo tipo de discapacidad es relevante, al generar un sentido de identidad y amistad que puede influenciar la satisfacción con la vida; (c) participar en actividades de rehabilitación y ocupacionales, como los huertos, la artesanía, la cocina brindan satisfacción con la vida y aportan a la identidad personal; (d) participar en deporte, como baloncesto, bocha o atletismo también es una fuente de bienestar físico y emocional; (e) se refiere bienestar personal al recibir apoyo específico como en temas de alimentación, apoyo económico, becas, transporte, terapias, consultas médicas, medicina e inserción educativa y laboral; (g) las personas con discapacidad que mencionan recibir apoyo, tienen más facilidades para aprovechar los recursos de las organización, es decir, utilizar de manera óptima las instalaciones, asistir a terapias físicas y psicológicas, cumplir con citas médicas y participar en actividades que la organización ofrece.

Para los familiares de personas con discapacidad se encontró que: (a) refieren la buena relación que hay con los trabajadores de las organizaciones y que recibir un trato cálido permite tener más confianza en los procesos; (b) las amistades que se forman con familiares de otras personas con discapacidad que asisten a las organizaciones ayudan a fortalecer las redes de apoyo y sentirse acompañados; (c) las madres refieren valorar más la vida, desde el reconocimiento que no todos la viven de la misma forma, desde la unión familiar y el apoyo mutuo; (d) las madres reflejan que ver a sus hijos e hijas desarrollando nuevas habilidades, teniendo aprendizajes hacia una vida con mayor independencia les da significado a sus vidas, reflejando deseos de estar allí cuando tengan logros; (e) algunas madres reconocen que el recibir apoyo para sus hijos representa apoyo para ellas, además, mencionan un cambio radical en su forma de ver la vida, con mayor compromiso con el futuro de sus hijos e hijas y el establecimiento de metas que representan sacrificios para apoyar en la independencia de sus familiares; (f) reconocer beneficios personales al participar en las organizaciones, está relacionado con el nivel en que los familiares se involucran en el proceso de los hijos e hijas, habiendo menos participación de padres que de madres.

Para los trabajadores se encontró que el trabajo con personas con discapacidad cambió su forma de relacionarse con la comunidad. También, teniendo en cuenta que la mayoría de los proyectos en los que trabajan son financiados a través de recaudación de fondos o donaciones, sus sueldos suelen ser percibidos como insuficientes, principalmente por la presencia de gastos necesarios que deben aportar a sus familias y en otros casos, los pagos o complementos de becas de estudios. A pesar de lo anterior, se identificó que la satisfacción que da el trabajo que realizan modera el afecto negativo que se puede generar ante tal per- 
cepción y el estrés laboral. Otro tema relacionado a la aceptación de los sueldos que reciben los trabajadores es la cantidad de aprendizajes y experiencias que se adquieren en el trabajo, elementos que no siempre se cubren en su formación universitaria.

\section{Discusión}

En cuanto al BS Ueda y Okawa (2003) manifiestan que es necesario considerar la experiencia subjetiva de las personas con discapacidad pues es información relevante para aportar a la evidencia en el tema de la discapacidad y con esto poder apoyar en a las comunidades hacia una mejor convivencia desde la inclusión. A partir del presente estudio se expande esta necesidad hacia los familiares y trabajadores de organizaciones de y para personas con discapacidad.

\section{BS de personas con discapacidad}

Se conoce que la forma de valorar cognitivamente el bienestar subjetivo en niveles altos modera los niveles de dolor físico cuando el caso aplique, pero también permite menores niveles de sintomatología depresiva (Furrer et al., 2019). Ese es el caso de las personas con discapacidad que participaron en el estudio, al observar los indicadores de BS en la Tabla 4 ( $68 \%$ con muy alta satisfacción con la vida, $32 \%$ con muy alto afecto positivo), los cuales se pueden profundizar con los hallazgos cualitativos, en donde se manifiestan diferentes experiencias que brindan sentido de identidad, como los nuevos retos o las relaciones con amistades cercanas. Apoyando estos resultados, Bouffard y Crocker (1992) plantean que para las personas con discapacidad, que la percepción de retos, como la consecución de metas personales se relaciona con alto afecto positivo, como se ve en la Tabla 4. A pesar de los altos niveles de satisfacción con la vida y afecto positivo, es importante tener en cuenta que existen personas que reportan altos niveles de afecto negativo ( $26 \%$ con alto afecto negativo) lo cual puede tener que ver con la severidad de la discapacidad, dinámica familiar o inclusión social.

La información cualitativa en las categorías "relación del apoyo por parte de familiares, amigos y trabajadores de las organizaciones con el bienestar personal" y "relación de participación en alguno de los servicios que brindan las organizaciones con el bienestar personal" brindan mayor evidencia a la aportada por Vera y colaboradores (2018) que indican que al- gunas variables asociadas a niveles altos de bienestar subjetivo en personas con discapacidad fueron el participar de las redes sociales y la confianza en amistades; mientras que los niveles bajos se relacionan con la falta de confianza en familiares y la falta de amistades.

Específicamente sobre la alta valoración que se brindó a la libertad y las nuevas experiencias por parte de las personas con discapacidad, se relaciona a los hallazgos de Hammel y colaboradores (2008) al explicar que las personas con discapacidad suelen manifestar la necesidad de ser libres para definir y buscar la participación deseada en sus propios términos, en lugar de cumplir con las normas sociales predeterminadas. Esto quiere decir que fomentar la libertad en las actividades que pueden hacer las personas con discapacidad influye positivamente en el bienestar, principalmente porque se vive en un contexto en donde los algunos cuidadores de personas con discapacidad tratan de mantenerlos en los hogares con la idea de protección y en otros casos, por prejuicios hacia la discapacidad, esto sucede principalmente si la persona con discapacidad es una mujer (Callus et al., 2019; Pettinicchio \& Maroto, 2017).

\section{BS de familiares de personas con discapacidad}

En el estudio se encontró que casi todos los familiares reportaron una alta o muy alta satisfacción con la vida $(66 \%$ con muy alta satisfacción con la vida, $56 \%$ con alto afecto positivo y $28 \%$ con bajo afecto negativo), esto puede tener que ver con que toda la muestra ya forma parte de los programas para personas con discapacidad en la región tz'utujil, que aunque no es un factor explicativo debido a las características del diseño de este estudio, es una característica de la muestra que la diferencia de las reportadas en otros estudios ( $\mathrm{Li}$ et al., 2015; van Campen \& van Santvoort, 2013).

Los resultados cualitativos reportados para familiares de personas con discapacidad, relacionados con el bienestar que produce el saber que sus hijas e hijos desarrollan nuevas habilidades, aprendizajes y logros, además del apoyo que reciben de las organizaciones fortalecen los hallazgos de Fekete y colaboradores (2019) quienes reportan que cuidadores de personas con discapacidad manifiestan mayor afecto positivo cuando perciben que sus familiares tienen mayor control en sus actividades.

Por otro lado, los niveles de afecto positivo en su mayoría son altos y pueden estar relacionados con el apoyo social percibido, como se reporta en el estudio de Halstead y colaboradores (2018). Con respecto a lo 
que reportan las madres sobre los beneficios personales que se reconocen en la participación de redes sociales de apoyo, se sabe que cuanto mayor sea el nivel de valoración positiva que las madres puedan identificar que la discapacidad infantil ha traído a la vida de la familia, por ejemplo, mejores valores familiares, sensibilidad hacia los demás en la comunidad, espiritualidad elevada, etc., mayor es el ajuste general de la familia (Trute et al., 2010), por ello, la información brindada por los familiares y los indicadores cuantitativos de BS son alentadores.

\section{BS de trabajadores de organizaciones para personas con discapacidad}

Los resultados cuantitativos de los trabajadores de las organizaciones se asemejan a los hallazgos de Mutkins y colaboradores (2011) al demostrar que la satisfacción con el trabajo es moderadora del cansancio laboral y lleva a interpretar que el bienestar de los trabajadores de las organizaciones se relaciona directamente con el bienestar de las personas con discapacidad. Además de ello, los resultados cualitativos brindan mayor comprensión a la importancia de la "esperanza disposicional" como un mediador del bienestar subjetivo, específicamente al observar que los participantes logran identificar elementos en medio de las dificultades que les permiten encontrar los beneficios personales a partir de su labor, reflexiones que según los hallazgos de (Oliver et al., 2019) pueden explicarse por una fuerte esperanza sobre la propia agencia para la consecución de objetivos.

\section{Reflexiones para todos los participantes del estudio}

Se ha reportado que en diferentes países, personas con discapacidad presentan desventajas en cuanto al BS en comparación con el resto de la población, y una de las variables que más aporta a estas brechas es el "apoyo social" (van Campen \& van Santvoort, 2013). Los resultados del presente estudio llevan a revisar la idea de que el posible mantenimiento de niveles altos de BS en los participantes del estudio, puede explicarse desde la teoría de la homeostasis del BS de Cummins y colaboradores (2009), esta propone que el bienestar subjetivo se controla y mantiene activamente, a través de un sistema denominado "homeostasis del BS" en las personas, el cual intenta mantener una visión positiva del yo en un nivel abstracto no específico. Este sistema actúa para proteger el afecto central, pero la capacidad de retener condiciones de bienestar dentro de un cierto rango de valores es limitada, por lo que circunstancias de vida adversas pueden sobrepasar esta capacidad y permita que los niveles de BS sean bajos. Pero en ese mismo sentido, es muy probable que la participación en redes sociales de apoyo o ser parte de organizaciones de y para personas con discapacidad, o de forma más general, la interacción y los vínculos entre los participantes de este estudio, favorezcan la homeostasis del BS.

Los hallazgos cualitativos en este estudio muestran la importancia que las personas con discapacidad y familiares dan a las amistades cercanas que brindan tratos dignos, lo cual es muy importante para retomar la necesidad de fomentar acciones para reducir el estigma hacia las personas con discapacidad y sus familiares, pues se sabe que el estigma es una fuerte influencia en los niveles bajos de BS (Werner \& Shulman, 2013).

Los resultados también demostraron que las personas con discapacidad y familiares presentan valores menores en afecto positivo, a comparación que los trabajadores y al compararlos se encontró una diferencia significativa entre los grupos, lo cual puede indicar aún las desventajas en cuanto al BS que tienen las personas con discapacidad y familiares con el resto de la población y representa un dato muy importante para seguir fortaleciendo las redes sociales de apoyo, la inclusión social y el acceso a recursos para personas con discapacidad y familiares.

Por último, la metodología y la temática explorada en esta investigación son relevantes para su seguimiento y mayor profundización, con poblaciones más específicas y robustas, y con ello poder avanzar en la investigación en la línea de salud mental y discapacidad en Guatemala y Centroamérica.

\section{Agradecimientos}

Se agradece a la Dirección General de Investigación de la Universidad de San Carlos de Guatemala por financiar la presente investigación a través de la partida presupuestaria 4.8.63.9.30 durante el año 2019 en el Programa Universitario de Investigación de Asentamientos Humanos. Se agradece a la Unidad de Investigación Profesional de la Escuela de Ciencias Psicológicas por avalar la investigación y los productos que de ella se generan. Así también, se agradece a Acopedis y a las cinco organizaciones del estudio por su apertura, interés y acompañamiento en el proceso 
de investigación. Se agradece a Paola Orellana, por su participación en la recolección de información cuantitativa y cualitativa del estudio y a Wendy Ortiz en su trabajo como auxiliar de investigación.

\section{Contribución de los autores}

Coordinación, elaboración y revisión del Documento: DS

Diseño de la recolección de datos o del trabajo en campo: DS, ER-C

Recolección o contribución de datos o realización del trabajo de campo: DS, ER-C

Limpieza, sistematización, análisis o visualización de datos: DS, ER-C

Participación en análisis de datos, estructura y en la escritura del documento: todos los autores

\section{Materiales suplementarios}

Los materiales suplementarios de este artículo se encuentran en la página web de la revista a través del doi:https://doi.org/10.36829/63CTS.v8i2.868

\section{Referencias}

Bouffard, M., \& Crocker, P. R. E. (1992). Coping by individuals with physical disabilities with perceived challenge in physical activity: Are people consistent? Research Quarterly for Exercise and Sport, 63(4), 410-417. https://doi.org/ 10.1080/02701367.1992.10608763

Callus, A. M., Bonello, I., Mifsud, C., \& Fenech, R. (2019). Overprotection in the lives of people with intellectual disability in Malta: Knowing what is control and what is enabling support. Disability and Society, 34(3), 345-367. https://doi.org/10.10 80/09687599.2018.1547186

Centro Internacional para Evidencia de Discapacidad. (2016). Informe de la II Encuesta Nacional de Discapacidad en Guatemala (ENDIS). London School of Hygiene and Tropical Medicine.

Cummins, R. A., Lau, A. A. L. D., Mellor, D., \& Stokes, M. A. (2009). Encouraging governments to enhance the happiness of their nation: Step
1: Understand subjective wellbeing. Social Indicators Research, 91(1), 23-36. https://doi.org/ 10.1007/s11205-008-9324-6

Diener, E., Emmons, R., Larsen, R., \& Griffin, S. (1985). The Satisfaction With Life Scale. Journal of Personality Assessment, 49(1), 71-75. https://doi.org/10.1207/s15327752jpa4901

Diener, E., Scollon, C. N., \& Lucas, R. E. (2009). The Evolving Concept of Subjective WellBeing: The Multifaceted Nature of Happiness. En E. Diener (Ed.), Assessing well-being: The collected works (pp. 67-100). Springer. https://doi.org/10.1007/978-90-481-2354-4_4

Diener, E., Suh, E. M., Lucas, R. E., \& Smith, H. L. (1999). Subjective well-being: Three decades of progress. Psychological Bulletin, 125(2), 276-302. https://doi.org/10.1037/0033-2909.125.2.276

Fekete, C., Tough, H., Brinkhof, M. W. G., \& Siegrist, J. (2019). Does well-being suffer when control in productive activities is low? A dyadic longitudinal analysis in the disability setting. Journal of Psychosomatic Research, 122(April), 13-23. https://doi.org/10.1016/j.jpsychores.2019.04.015

Furrer, A., Michel, G., Terrill, A. L., Jensen, M. P., \& Müller, R. (2019). Modeling subjective well-being in individuals with chronic pain and a physical disability: The role of pain control and pain catastrophizing. Disability and Rehabilitation, 41(5), 498-507. https://doi.org/10.1080/0963828 8.2017.1390614

Halstead, E. J., Griffith, G. M., \& Hastings, R. P. (2018). Social support, coping, and positive perceptions as potential protective factors for the well-being of mothers of children with intellectual and developmental disabilities. International Journal of Developmental Disabilities, 64(4-5), 297-305. https://doi.org/10.1080/20473869.2017.1329192

Hammel, J. O. Y., Magasi, S., Heinemann, A., Whiteneck, G., Bogner, J., \& Rodriguez, E. (2008). What does participation mean ? An insider perspective from people with disabilities. Disability and Rehabilitation, 30(19), 1445-1460. https://doi.org/10.1080/09638280701625534

Instituto Nacional de Estadística. (2020). Base de datos CENSO 2018 [Base de datos]. Autor. https://www.censopoblacion.gt/descarga 
Joiner, T., Sandin, B., Chorot, P., Lostao, L., \& Marquina, G. (1997). Development and Factor Analytic Validation of the SPANAS Among Women in Spain. Journal of Personality Assessment, 68(3), 508-531. https://doi.org/ 10.1207/s15327752jpa6803

Li, A., Shaffer, J., \& Bagger, J. (2015). The psychological well-being of disability caregivers: Examining the roles of family strain, family-to-work conflict, and perceived supervisor support. Journal of Occupational Health Psychology, 20(1), 40-49. https://doi.org/10.1037/a0037878

Mutkins, E., Brown, R. F., \& Thorsteinsson, E. B. (2011). Stress, depression, workplace and social supports and burnout in intellectual disability support staff. Journal of Intellectual Disability Research, 55(5), 500-510. https://doi.org/10.1111/ j.1365-2788.2011.01406.x

Oliver, A., Revuelto, L., Fernández, I., Simó-Algado, S., \& Galiana, L. (2019). An integrative model of the subjective well-being of staff working in intellectual disability services. Research in Developmental Disabilities, 87, 1-8. https://doi.org/ 10.1016/j.ridd.2019.01.007

Pavot, W., \& Diener, E. (1993). Review of the Satisfaction With Life Scale. Psychological Assesment, 5(2), 164-172. https://doi.org/10.1007/978-90-4812354-4 5

Pettinicchio, D., \& Maroto, M. (2017). Employment outcomes among men and women with disabilities: How the intersection of gender and disability status shapes labor market inequality. En Factors in Studying Employment for Persons with Disability (Research in Social Science and Disability, Vol. 10, pp. 3-33). ). Emerald. https://doi.org/10.1108/S1479-354720170000010003

Roelen, K., \& Camfiel, L. (2015). Mixed Methods Research in Poverty and Vulnerability. Palgrave Macmillan.
Trute, B., Benzies, K. M., Worthington, C., Reddon, J. R., \& Moore, M. (2010). Accentuate the positive to mitigate the negative: Mother psychological coping resources and family adjustment in childhood disability. Journal of Intellectual and Developmental Disability, 35(1), 36-43. https://doi.org/10.3109/13668250903496328

Ueda, S., \& Okawa, Y. (2003). The subjective dimension of functioning and disability: What is it and what is it for? Disability and Rehabilitation, 25(11-12), 596-601. https://doi.org/ 10.1080/0963 828031000 137108

van Campen, C., \& van Santvoort, M. (2013). Explaining Low Subjective Well-Being of Persons with Disabilities in Europe: The Impact of Disability, Personal Resources, Participation and Socio-Economic Status. Social Indicators Research, 111(3), 839-854. https://doi.org/10.1007/ s11205-012-0036-6

Vera, J., Tánori, J., Bautista, G., \& Rodríguez, C. (2018). Bienestar subjetivo en personas con discapacidad motriz en México. Revista Psicologia e Saúde, 10(2), 15-24. https://doi.org/10.20435/pssa. v9i1.375

Watson, D., Clark, L. A., \& Tellegen, A. (1988). Development and Validation of Brief Measures of Positive and Negative Affect: The PANAS Scales. Journal of Personality and Social Psychology, 54(6), 1063-1070. https://doi.org/10.1037/00223514.54.6.1063

Werner, S., \& Shulman, C. (2013). Subjective wellbeing among family caregivers of individuals with developmental disabilities: The role of affiliate stigma and psychosocial moderating variables. Research in Developmental Disabilities, 34(11), 4103-4114. https://doi.org/10.1016/j.ridd. 2013.08.029 